\begin{tabular}{ccc}
\hline International Journal of Engineering \& Technology, $7(2.24)(2018) 231-234$ \\
International Journal of Engineering \& Technology \\
SPC \\
Website: www.sciencepubco.com/index.php/IJET \\
Research paper
\end{tabular}

\title{
Mine Detecting Robot with Multi Sensors Controlled Using HC-12 Module
}

\author{
Jebasingh Kirubakaran.S.J' ${ }^{1}$ Anish kumar jha², Dheeraj kumar³, Sadambi Poornachandran Prakash ${ }^{4}$ \\ ${ }^{1}$ Assistant Professor, ${ }^{2,3,4}$ UG Scholar \\ 1,2,3,4 Department of Electronics and Communication Engineering, \\ Vel Tech Multi Tech Dr. Rangarajan Dr.Sakunthala Engineering College, Avadi, Chennai, India \\ *Corresponding Author E-mail: jebasinghvmt@gmail.com
}

\begin{abstract}
Application of robot for person safekeeping is an important aspect in the explore province. From medical to military use several functions are residential to fructify person life. A mine detecting robot is a wide military progression and a lifesaving design that can benefit humankind. The robot is guided by HC-12 module that allows it to scan the testing area within $1.8 \mathrm{~km}$ and metal detector is used as mine detecting sensor placed in front of the vehicle, while GPS is used to provide the exact location of the infected area. This paper put forward to design of a mine detecting sensor using HC-12 and GPS while applying it in a robotic sample. The embedded proposed system is based on Arduino technologies and guided by an HC -12 Module.
\end{abstract}

Keywords- Robot; Sensor; Security Detection; Wireless Communication

\section{Introduction}

There are several aspects like mechanics, electronics, computers and programming which are involved in Robotics. During the last century there a big progress in the robotics field, robotics has a wide range of applications in human lives. Various fields such as military, security, medical etc employ robots for accomplishing the task.

In military and security purpose a robot can do a work that humans are not willing to do and can be applied in remote operation, remote medical treatment, remote monitoring in dangerous environments.

Mine Detecting Robot Based on Wireless Communication with Multi-sensor Robot based on wireless communication with multisensor, which can carry out robot's motion control, video surveillance, temperature and humidity detection, gas detection and data exchange, etc. In this paper, utilize of robots in the mine recognition field from distance can improve gigantically (up-to $1 \mathrm{~km}$ ) using a HC-12 module. Another improvement is finding the exact location of mine detected place and sending the location of the infected place to the concerned persons using HC12 module. The robot trial product is based on Arduino technologies forbidden by an HC-12 module using PC is capable of detecting mines using Metal Detector. The robot consists of various components like Ultrasonic sensor, Metal Sensor, GPS, LCD screen, Motor drivers.

\section{Basic Components and Control Principle of the System}

The hardware parts of the robot mainly include four ultrasonic sensors, metal sensors, GPS (Global Positioning Satellite), HC-12 module, two DC motors, robot mechanism, and LCD and audrino mega board with power supply.

The control section consists of audrino Uno, HC-12 and a PC.

\subsection{Working principle}

This system is controlled by arduino controller. When the robot is connected arduino interface by the use of HC-12 module, and the Arduino electronic Board makes the scanning operation purposeful, while the robot starts receiving a steering control order. And it has connected with some external sensors like humidity and temperature sensor's so we can monitor the mine exact status of temperature level and humidity level.

When the robots detect a mine it will send a "Mine detected" message to the concerned persons along with the exact location of the mine detected. The mine detected message and location is sent via Hc-12 Module. "Mine detected" message will be shown in the LCD screen present on the robot. The robot has also four ultrasonic sensors which will guide the robot according to the given conditions as per the program. 


\section{Block Diagram}

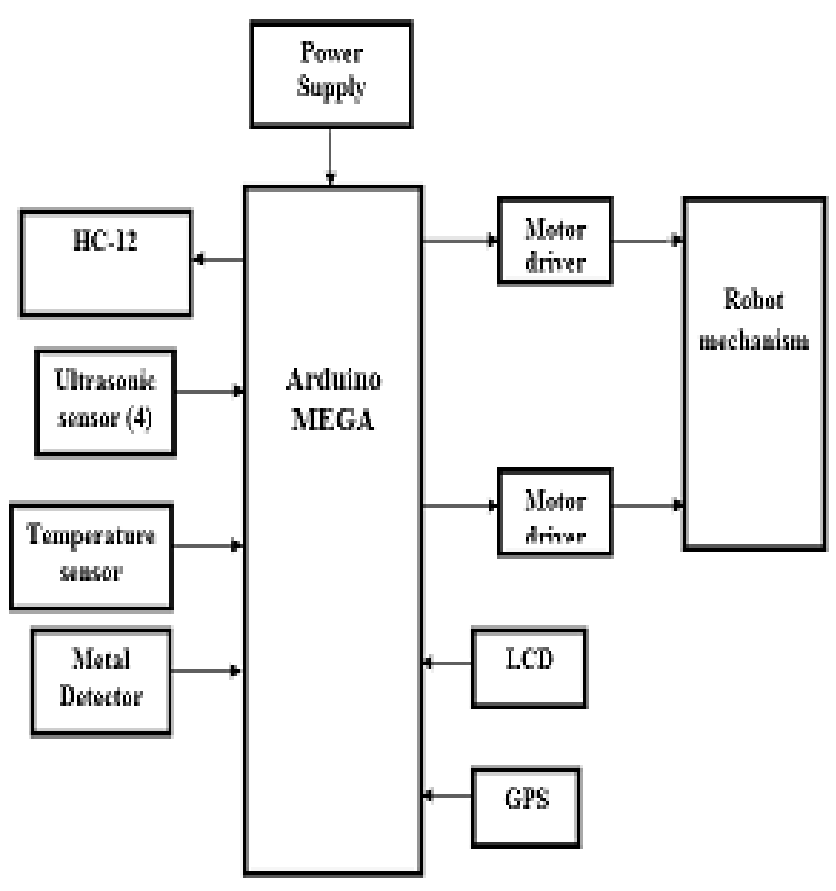

Fig. 1: Block diagram

\section{Control Section}

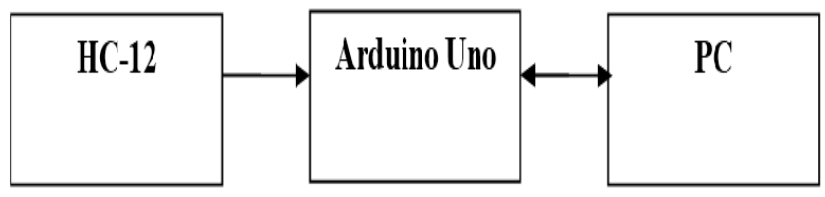

Fig. 2: Control section

\section{Hardware Implementation}

The hardware require in mine detecting robot is given asd follows:
A. AUDRINO MEGA BOARD
B. ROBOT MECHANISM
C. TEMPRATURE SENSOR
D. ULTARSONIC SENSOR
E. HC-12 MODIULE
F. GPS

\subsection{Movement Control of Mine Detecting Robot}

The designed robot is an autonomous robot and therefore the control of the robot is an important part to discuss. The autonomous robot consists of ultrasonic sensors for obstacle detection which is the first condition for the movement of vehicle. While in moving if an obstacle is detected it changes its direction else keeps the same track of motion. In the designed robot there are 4 ultrasonic sensors placed at front and back of the vehicle which decides the movement of the vehicle. Each movement is described below.

\subsection{Robot moving in the Left direction}

In order to move the autonomous vehicle, the command has to be given through serial monitor from PC. Once the start command is given the autonomous vehicle a start moving in forward direction i.e. the dc motor rotates in clockwise direction. To make the robot move in the left direction a command is given such as left arrow key through serial monitor. Once the data is obtained by the
Aurdino board the left dc motor stops running while the other motor runs continuously in clockwise direction.

The robot keeps on moving in the left direction until the command is changed or an obstacle is detected. If an obstacle is detected the direction of robot changes accordingly. The obstacle is detected by the front left ultrasonic sensor placed on the body of the robot. The Vcc and ground terminal of this sensor is connected to a separate chip. In this separate chip 2 rows are soldered one as Vcc and other as ground. The echo pin of the front left sensor is connected to the $3^{\text {rd }}$ pin of atmega board while the trigger pin of this sensor is connected to the $2^{\text {nd }}$ pin of at mega board.

The direction in which the autonomous vehicle is moving is displayed on the serial monitor and also the LCD connected to the setup.

\subsection{Robot moving in the Right direction}

To make the robot move in the right direction a command is given such as right arrow key. Once the data is obtained by the Aurdino board, the right dc motor stops running while the other motor runs continuously in clockwise direction.

The robot keeps on moving in the right direction until the command is changed or an obstacle is detected or a metal is detected. If an obstacle is detected the direction of robot changes accordingly while if a metal is detected the robot stops instantly and provides the location of the infected place which is discussed later in the chapter. The obstacle is detected by the front right ultrasonic sensor placed on the body of robot. The vec and ground terminal of this sensor is connected to a separate chip. In this separate chip 2 rows are soldered one as vcc and other as ground. The echo pin of the front left sensor is connected to the $5^{\text {th }}$ pin of atmega board while the trigger pin of this sensor is connected to the $4^{\text {th }}$ pin of Atmega board.

The direction in which the autonomous vehicle is moving is displayed on the serial monitor and also the LCD connected to the setup.

\subsection{Robot Moving in Forward Direction}

To make the robot move in the forward direction a command is given such as top arrow key. Once the data is obtained by the Aurdino board, both the dc motor runs in clockwise direction. The possibilities of robot moving in forward direction also arise when the backward placed sensors are giving high digital output.

The robot keeps on moving in the forward direction until the command is changed or an obstacle is detected or metal is detected. If an obstacle is detected the direction of robot changes accordingly while if a metal is detected the robot stops instantly and provides the location of the infected place which is discussed later in the chapter. Here the obstacle is detected by the forward placed sensors on robot. If the obstacle is detected on both the sensors then the robot starts moving in backward direction automatically. The direction in which the autonomous vehicle is moving is displayed on the serial monitor and also the LCD connected to the setup.

\subsection{Robot Moving in Backward Direction}

To make the robot move in the backward direction a command is given such as left arrow key, once the data is obtained by the Aurdino board, both the dc motors run in anticlockwise direction continuously. The possibilities of robot moving in backward direction also arise when the forward placed sensors are giving high digital output.

The robot keeps on moving in the backward direction until the command is changed, an obstacle is detected or metal is detected. If an obstacle is detected the direction of robot changes accordingly while if a metal is detected the robot stops instantly and provides the location of the infected place which is discussed later in the chapter. 
Table 1: Control Mechanism of Robot

\begin{tabular}{|l|l|}
\hline Command & Movement \\
\hline Top Arrow Key & Forward Direction \\
\hline Bottom Arrow Key & Backward Direction \\
\hline Left Arrow Key & Left Direction \\
\hline Right Arrow Key & Right Direction \\
\hline 0 & Stop \\
\hline
\end{tabular}

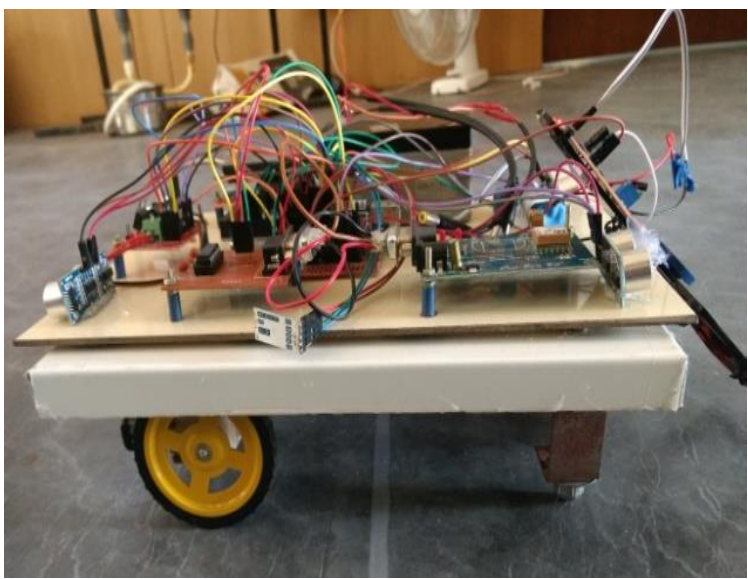

Fig. 3: Mine detection robot side view

Here the obstacle is detected by the backward placed sensors on robot. If the obstacle is detected on both the sensors then the robot starts moving in forward direction automatically. The direction in which the autonomous vehicle is moving is displayed on the serial monitor and also the LCD connected to the setup.

\section{Detection of Land Mines}

After knowing the movement mechanism of autonomous vehicle the detection of mines through metal detector is studied. The metal detector is placed in front of the vehicle in an inclined position. The supply is given to the metal detector using a 9v battery. The signal of metal detection is send to ATmega board at TX3 14 pin. The instant when metal is detected the autonomous vehicle stops and provides the location of detected mine which is done by GPS. The output is obtained on the serial monitor of metal detection and also the LCD connected in the setup. The LCD also displays the data like temperature of the current location.

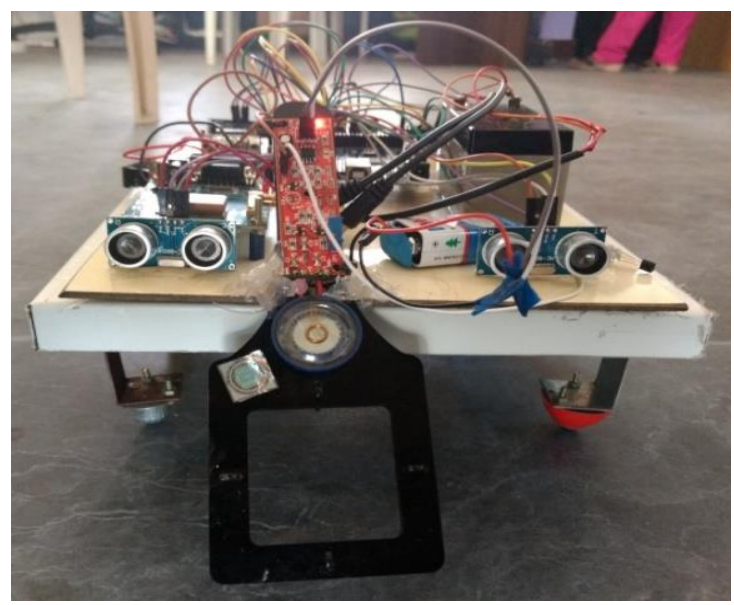

Fig. 4: Mine detecting robot front view with metal sensor

\section{Acquisition of Infected Place}

Once the robot reaches the location of infected area, it comes to halt position. The location of the infected area is given through
GPS sensor. The GPS sensor contains an antenna to get the longitude and latitude value.

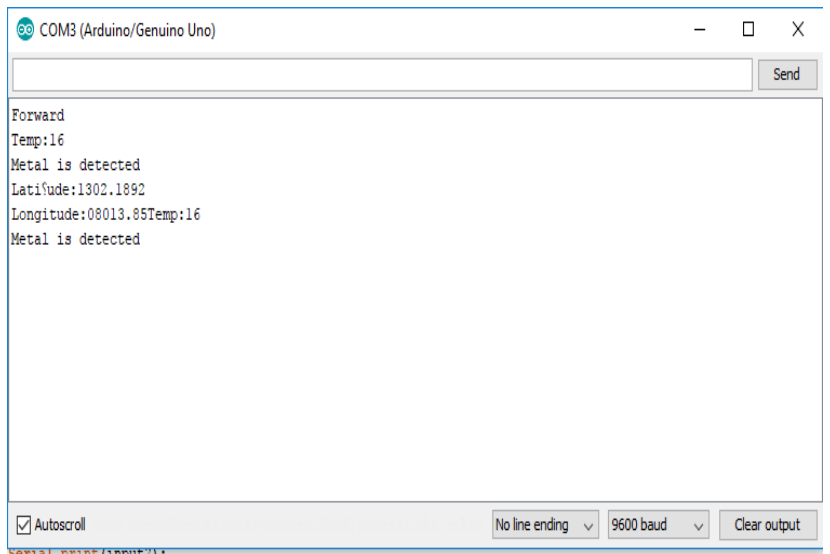

Fig. 5: Display of location

The serial communication of GPS sensor to ATmega board is done through a TTL logic containing MAX232 IC. Therefore once the location of infected place is obtained the robot can be moved further for searching of mines and the infected place can be avoided during human operation.

\section{Software Control}

Robot software control consists of two parts: detection control and robot driving control. Detection control consists of metal detector which senses the mines and to detect the corresponding infected place location using GPS and send to the appropriate persons.

Robot walking is mainly controlled by robot drive motor, consisting of two DC motors, which can realize forward, backward, left and right walks. Robot is programmed by using HC-12 module for its navigation and four ultrasonic sensors are attached to it.

\section{Results}

Results are obtained in the Serial monitor screen. The robot continuosly sends the status of the environment i.e., temperature and mine detection status. When the mine is detected the robot send mine detected message along with the GPS location (latitude and longitude).

\section{Conclusion and Future Work}

At present, robot is increasingly applied in security field. Continuous development of robot technology is expanding robot's application to various fields. Here the mine detecting prototype is designed an robotic vehicle which can sense metals near to it on its track and this robot is controlled by an HC-12 module application. In addition to this ultrasonic sensors are used for detecting the obstacles in various directions. Based on the hindrances present, the mine detecting robot takes alternate paths. In future we can also use the camera for better images of buried mines. Another future advancement in this prototype may include Shock absorbers and adjusters that can be installed to the wheels, so that the robot can run on any terrain. 


\section{References}

[1] "Mine detecting robot prototyping" Chamakh Youssef; Ben Rached Mohamed; Abdennour Najmeddine Tarek Frikha; Wassim Jmal 2017 2nd International Conference on Anti-Cyber Crimes (ICACC) Year: 2017 Pages: $183-186$

[2] "Autonomous defense robot in metal detecting and chemical sensing using internet of things" M. Yuvaraju, Drishya.C International Journal of Advanced Research in Electrical, Electronics and Instrumentation Engineering; Vol. 6, Issue 6, June 2017

[3] "Mine detecting robot based on wireless communication with multi sensor" Zhenjun He; Jiang Zhang; Peng Xu; Jiaheng Qin; Yunkai Zhu2013 IEEE 4th International Conference on Electronics Information and Emergency Communication Year: 2013 Pages: $117-120$

[4] "Strategy and development of mine detection robot system" Yeonsub Jin; Deokhyun Ko; Jushin Seok; Woosub Lee; Sungchul Kang2013 13th International Conference on Control, Automation and Systems.

[5] "Detecting robot system for mine disasters" Zhao-long Xu; Jie Yang; Shan Cai; Xiao-zhi Liu2010 International Conference on EProduct E-Service and E-Entertainment Year: 2010 Pages: 1 - 4.

[6] S.V.Manikanthan and K.srividhya "An Android based secure access control using ARM and cloud computing", Published in: Electronics and Communication Systems (ICECS), 2015 2nd International Conference on 26-27 Feb. 2015, Publisher: IEEE, DOI: 10.1109/ECS.2015.7124833.

[7] T. Padmapriya and V. Saminadan, "Priority based fair resource allocation and Admission Control Technique for Multi-user Multiclass downlink Traffic in LTE-Advanced Networks", International Journal of Advanced Research, vol.5, no.1, pp.1633-1641, January 2017. 